\title{
Large effect of polydispersity on defect concentrations in colloidal crystals
}

\author{
Sander Pronk and Daan Frenkel \\ FOM Institute for Atomic and Molecular Physics \\ Kruislaan 407 \\ 1098 SJ Amsterdam \\ the Netherlands
}

\begin{abstract}
We compute the equilibrium concentration of stacking faults and point defects in polydisperse hard-sphere crystals. We find that, while the concentration of stacking faults remains similar to that of monodisperse hard sphere crystals, the concentration of vacancies decreases by about a factor two. Most strikingly, the concentration of interstitials in the maximally polydisperse crystal may be some six orders of magnitude larger than in a monodisperse crystal. We show that this dramatic increase in interstitial concentration is due to the increased probability of finding small particles and that the small-particle tail of the particle size distribution is crucial for the interstitial concentration in a colloidal crystal.
\end{abstract}

\section{INTRODUCTION}

The experimental study of colloidal crystals is of interest for at least two reasons. First of all, the possibility to design the constituents of such crystals, allows us to gain insight into the factors that determine the structure and kinetics of formation of crystalline materials. In addition, colloidal crystals are of interest because of their potential application as photonic materials[1]. To a first approximation, one might view colloidal crystals as scale models of atomic crystals. But this analogy is flawed for several reasons. First of all, the intermolecular forces between colloidal particles may be qualitatively different from those between atoms. Secondly, the dynamics of colloidal matter is intrinsically different from that of atomic materials, due to the presence of a solvent. Finally, unlike atomic materials, colloidal systems are never completely monodisperse. This polydispersity may have important consequence for the phase behavior and structural properties of the colloidal crystals. In addition, polydispersity can have an effect on the equilibrium concentration of (point) defects in colloidal crystals. As defects may strongly influence the photonic properties of colloidal crystals, a better understanding of the effect of polydispersity on defect concentrations, may also be of practical relevance for the design of photonic crystals.

In the present paper, we describe a numerical study of the effect of polydispersity on the concentration of stacking faults, vacancies and interstitials in hard-sphere colloidal crystals.

\section{SIMULATION METHODS}

\section{A. Semigrand Canonical Ensemble}

To simulate a the equilibrium properties of polydisperse hard-sphere crystals, we used the semigrand canonical ensemble method [2, 3]. For a system with continuous size polydispersity, the free-energy functional of the semigrand canonical ensemble is given by:

$$
\begin{array}{r}
Y\left(N, P, T, \sigma_{0},\{\Delta \mu\}\right)=U-T S+P V+N \mu\left(\sigma_{0}\right) \\
-N \int d \sigma\left[\mu(\sigma)-\mu\left(\sigma_{0}\right)\right] p(\sigma)
\end{array}
$$

where $N$ is the total number of particles in the system, $P$ is the pressure, $T$ is the temperature and the set $\{\Delta \mu\}$ denotes the differences between $\mu(\sigma)$, the chemical potential of a species with diameter $\sigma$, and $\mu\left(\sigma_{0}\right)$, the chemical potential of an (otherwise arbitrary) reference species: $\Delta \mu(\sigma) \equiv \mu(\sigma)-\mu\left(\sigma_{0}\right)$. As we are dealing with hard-core particles, we choose our unit of energy to be equal to $k_{B} T . p(\sigma)$ denotes the probability of finding a particle with diameter $\sigma$. The set of thermodynamic fields $\{\Delta \mu\}$ act as control parameters that determine the particle-size distribution. In the present work, we assume a quadratic dependence of $\Delta \mu(\sigma)$ on $\sigma-\sigma_{0}$ :

$$
\beta\left[\mu(\sigma)-\mu\left(\sigma_{0}\right)\right]=-\left(\sigma-\sigma_{0}\right)^{2} / 2 v
$$

Where $\beta \equiv 1 / k_{B} T$. The parameter $v$ determines the degree of polydispersity. At infinite dilution, the size distribution is directly given by $p(\sigma)=c \exp \left(-\left(\sigma-\sigma_{0}\right)^{2} / 2 v\right)$. At finite concentrations, the size distribution cannot be inferred directly from the functional form of $\Delta \mu(\sigma)$. Both the average particle diameter and the actual polydispersity $s$ (defined through $\left.s^{2} \equiv\left\langle\sigma^{2}\right\rangle /\langle\sigma\rangle^{2}-1\right)$ must be determined in the semigrand ensemble simulations. Once the functional form of $\Delta \mu(\sigma)$ has been specified, the semi-grand partition function $\Xi$ is a function of $N, P, T, v$ and $\sigma_{0}$.

$$
\begin{array}{r}
\Xi\left(N, P, T, v, \sigma_{0}\right)= \\
\int d V \int d \mathbf{r}^{N} \int d \sigma^{N} \exp \left(-\beta\left[P V+U\left(r^{N}, \sigma^{N}\right)\right]\right. \\
\left.-\sum_{i} \frac{\left(\sigma_{i}-\sigma_{0}\right)^{2}}{2 v}\right)
\end{array}
$$

The semigrand free energy $Y$ is related to $\Xi$ through $Y=$ $-k_{B} T \ln \Xi$. To sample the configurations of the semi-grand ensemble, we use Metropolis-style Monte Carlo sampling of all variables that characterize a given configuration of the $N$ particle system. In addition to the usual trial moves that attempt to change the particle coordinates $\left\{\mathbf{r}^{N}\right\}$ and the system volume $V$, there are trial moves to change the diameter of a particle. As has been explained by Bolhuis and Kofke, it is computationally more efficient to combine volume-changing moves with particle resizing moves [3].

To calculate the chemical potential of the reference species, thermodynamic integration was used. As a reference state, we took the monodisperse hard-sphere crystal near coexistence, 
for which the free energy per particle is accurately known [4]. In order to compute the change in free energy with $P$ and $v$, we make use of the following thermodynamic relations:

$$
\begin{aligned}
& \left(\frac{\partial Y}{\partial P}\right)_{N, T, \sigma_{0}, v}=V \\
& \left(\frac{\partial Y}{\partial v}\right)_{N, P, T, \sigma_{0}}=N \int d \sigma^{\prime} p\left(\sigma^{\prime}\right) \frac{-\left(\sigma^{\prime}-\sigma_{0}\right)^{2}}{2 v^{2}}
\end{aligned}
$$

The semigrand free energy of an ideal, non-interacting system of polydisperse particles, is

$$
\begin{aligned}
Y_{\mathrm{id}}= & -k_{B} T \ln \int d V \exp (-\beta P V) \\
& \int d \mathbf{r}^{N} \int d \sigma^{N} \exp \left(-\sum_{i} \frac{\left(\sigma_{i}-\sigma_{0}\right)^{2}}{2 v}\right) \\
= & N \mu_{\mathrm{id}}\left(\sigma_{0}\right)=G_{\mathrm{id}}-\frac{N k_{B} T}{2} \ln (2 \pi v)
\end{aligned}
$$

We can now employ the following scheme to compute $\mu_{\mathrm{ex}}\left(\sigma_{0}\right)$ by thermodynamic integration, using as input our knowledge of the excess chemical potential $\mu_{\mathrm{ex}, 0}$ of a monodisperse hard sphere system at pressure $P_{0}$ :

$$
\begin{aligned}
\mu_{\mathrm{ex}}\left(\sigma_{0}\right)= & \mu_{\mathrm{ex}, 0}+\frac{1}{N} \int_{P_{0}}^{P} d P^{\prime}\left\langle V-\frac{(N+1) k_{B} T}{P^{\prime}}\right\rangle \\
& +\frac{1}{N} \int_{0}^{v} d v^{\prime}\left\langle\frac{-\sum_{i}\left(\sigma_{i}-\sigma_{0}\right)^{2}}{2 v^{\prime 2}}+\frac{N k_{B} T}{2 v^{\prime}}\right\rangle
\end{aligned}
$$

\section{B. Interstitial Concentration}

The methods that we used to calculate the concentration of point defects are similar to those discussed in Ref. 5. We first consider the free energy $Y_{M, n_{V}, n_{I}}$ of a crystalline system containing $M$ lattice sites, $n_{V}$ vacancies and $n_{I}$ interstitials. The total number of particles in this system is $N=M+n_{I}-n_{V}$. It is convenient to consider interstitials and vacancies separately.

By analogy to the derivation of interstitial concentrations in monodisperse systems[5], it is straightforward to show that the concentration of interstitials $\left(x_{I}\right)$ is given by $x_{I} \approx$ $\exp \left(-\beta y_{I}\right)$, where $y_{I}$ is defined as $y_{I}=Y_{M, 0,1}-Y_{M+1,0,0}$. It is convenient to rewrite $y_{I}$ as

$$
\begin{aligned}
y_{I} & =Y_{M, 0,1}-Y_{M+1,0,0} \\
& =Y_{M, 0,1}-Y_{M, 0,0}+Y_{M, 0,0}-Y_{M+1,0,0} \\
& =Y_{M, 0,1}-Y_{M, 0,0}-\left[\mu_{\mathrm{id}}\left(\sigma_{0}\right)+\mu_{\mathrm{ex}}\left(\sigma_{0}\right)\right] \\
& =Y_{M, 0,1}-\left[Y_{M, 0,0}+\mu_{\mathrm{id}}\left(\sigma_{0}\right)\right]-\mu_{\mathrm{ex}}\left(\sigma_{0}\right) \\
& =y_{\mathrm{add}}-\mu_{\mathrm{ex}}\left(\sigma_{0}\right)
\end{aligned}
$$

Here $y_{\text {add }}$ is the free energy difference between a system with one interstitial and a perfect crystal plus one ideal (noninteracting) particle. The quantity $Y_{M+1,0,0}$, the free energy of a system with $M+1$ lattice sites and no defects, is an abstract quantity that does not neccesarily correspond to a crystal with realizable lattice in an orthorhombic simulation box; its value is well-defined by virtue of the extensivity of free energy.

To calculate $y_{\text {add }}$, we simulate a crystal with $M$ lattice sites and $M+1$ particles, of which particle $j$ has a scaled hard-core diameter $a \sigma_{j}$. The diameter scaling parameter $a$ can be varied during the simulation, so that we sample the partition defined by

$$
\begin{aligned}
& \Xi_{M, 0,1}^{\prime}\left(M+1, P, T, \sigma_{0}, \nu\right)= \\
& \quad \int_{0}^{1} d a \Xi_{M, 0,1}\left(M+1, P, T, \sigma_{0}, \nu, a\right)
\end{aligned}
$$

where $\Xi_{M, 0,1}\left(M+1, P, T, v, \sigma_{0}, a\right)$ is defined as in Eq. 3 but with configurational energy $U\left(\mathbf{r}^{M}, \sigma^{M}, a \sigma_{j}\right)$. We stress that particle $j$ differs from the other particles only in the overlap criterion, not in the probability distribution that determines diameter sampling: for the overlap criterion, the particle radius of this particle is $a \sigma$, whereas its weight in the Semigrand chemical potential distribution of Eq. 2 is still determined by $\sigma$.

During the simulation, we construct a histogram $P(a \mid M+$ $1, P, T, v)$ :

$$
\begin{aligned}
& P(a \mid M+1, P, T, \vee)= \\
& \frac{\int_{0}^{1} d a^{\prime} \delta\left(a-a^{\prime}\right) \Xi_{M, 0,1}\left(M+1, P, T, \vee, \sigma_{0}, a\right)}{\Xi_{M, 0,1}^{\prime}\left(M+1, P, T, \nu, \sigma_{0}\right)}
\end{aligned}
$$

With this histogram we can calculate

$$
y_{\text {grow }}=-k_{B} T \ln \frac{P\left(a=1 \mid M+1, P, T, \nu, \sigma_{0}\right)}{P\left(a=0 \mid M+1, P, T, \nu, \sigma_{0}\right)}
$$

where $y_{\text {grow }}$ is the reversible work needed to transform an interacting point particle $(a=0)$ into a particle with a hard-core diameter $\sigma_{j}$ (corresponding to $a=1$ ). In order to sample the full range of $a$-values from 0 to 1 , it is necessary to use biased sampling. We employed multicanonical/umbrella sampling [6, 7] to generate $P\left(a \mid M, P, T, v, \sigma_{0}\right)$.

To obtain the total interstitial free energy $y_{\text {add }}$ we must still add the free energy change associated with the transformation of a non-interacting particle into an interacting point particle. This free energy change is determined by the ratio of the volumes accessible to the two types of particles:

$$
\begin{aligned}
y_{\text {add }}-y_{\text {grow }} & =-k_{B} T \ln \frac{\left\langle V_{\text {acc }}\right\rangle}{V} \\
& =-k_{B} T \ln \langle 1-\eta\rangle
\end{aligned}
$$

where $V_{\text {acc }}$ is the volume accessible to the point particle and $\eta$ denotes the volume fraction of the defect-free hard-sphere crystal. It is not necessary to confine the interstitial to a particular Wigner-Seitz cell, as interstitials diffuse quickly through the system. If this were not the case, both the scaled and the unscaled particle would have to be confined to a particular Wigner-Seitz cell (or even, to one particular interstitial cavity). 


\section{Vacancy Concentration}

For the vacancies, we can get for the concentration $x_{V} \approx$ $\exp \left(-\beta y_{V}\right)$ (see Ref. 5), with $y_{V}=Y_{M+1,1,0}-Y_{M, 0,0}$ and introduce the analogous free energy to $y_{\text {add }}$ :

$$
\begin{aligned}
y_{V} & =Y_{M+1,1,0}-Y_{M, 0,0} \\
& =Y_{M+1,1,0}-Y_{M+1,0,0}+Y_{M+1,0,0}-Y_{M, 0,0} \\
& =Y_{M+1,1,0}-Y_{M+1,0,0}+\mu\left(\sigma_{0}\right) \\
& =Y_{M+1,1,0}-Y_{M+1,0,0}+\mu_{\mathrm{id}}\left(\sigma_{0}\right)+\mu_{\mathrm{ex}}\left(\sigma_{0}\right) \\
& =-\left(Y_{M+1,0,0}-\left[Y_{M+1,1,0}+\mu_{\mathrm{id}}\left(\sigma_{0}\right)\right]\right)+\mu_{\mathrm{ex}}\left(\sigma_{0}\right) \\
& =-y_{\mathrm{rem}}+\mu_{\mathrm{ex}}\left(\sigma_{0}\right)
\end{aligned}
$$

In this case, $y_{\text {rem }}$ is the free energy difference between a perfect crystal and a crystal with one vacancy plus a noninteracting particle.

If we assume that we can sample a system which can switch one particle between being a normal particle $\left(b=b_{n}\right)$ and a non-interacting particle $\left(b=b_{i}\right)$, we can introduce the equilibrium probability $P\left(b \mid M, P, T, \vee, \sigma_{0}\right)$ :

$$
\begin{aligned}
y_{\text {rem }} & =-k_{B} T \ln \frac{P\left(b_{n} \mid M, P, T, \nu, \sigma_{0}\right)}{P\left(b_{i} \mid M, P, T, \nu, \sigma_{0}\right)} \\
& =-k_{B} T \ln \frac{\left\langle\pi\left(b_{i} \rightarrow b_{n}\right)\right\rangle}{\left\langle\pi\left(b_{n} \rightarrow b_{i}\right)\right\rangle}
\end{aligned}
$$

where $\left\langle\pi\left(b_{i} \rightarrow b_{n}\right)\right\rangle$ is the mean transition probability from $b=b_{i}$ to $b=b_{n}$. Because a real particle can always switch to a non-interacting, particle, we can reduce the expression for $y_{\text {rem }}$ to

$$
y_{\mathrm{rem}}=-k_{B} T \ln \left\langle\pi\left(b_{i} \rightarrow b_{n}\right)\right\rangle
$$

Now $\left\langle\pi\left(b_{i} \rightarrow b_{n}\right)\right\rangle$, the transition probability from a state of a system with a vacancy and a non-interacting particle to a perfect crystal, is related to the probability $P_{\text {ins }}$ for the insertion of a (normal polydisperse) particle into the vacancy:

$$
-k_{B} T \ln \left\langle\pi\left(b_{i} \rightarrow b_{n}\right)\right\rangle=-k_{B} T\left(\ln P_{\mathrm{ins}}\right)
$$

In practice, the simulation will consist of a collection of $M-1$ normal particles and one ideal polydisperse particle which we keep in the Wigner-Seitz cell of the tracked vacancy. We then do multicanonical sampling, biasing on the number of overlaps that the ideal particle would create if it would be switched to a real particle, and get $P_{\text {ins }}$ from the probability to create zero overlaps. This scheme is essentially identical to that of Bennett and Alder[8], save for the multicanonical sampling.

\section{RESULTS}

The simulations to calculate the point defect concentration were done at various points along the melting line of polydisperse hard sphere crystals, as taken from Ref. 3. The points chosen give a polydispersity of approx. $1.5 \%, 3 \%, 5 \%$ and

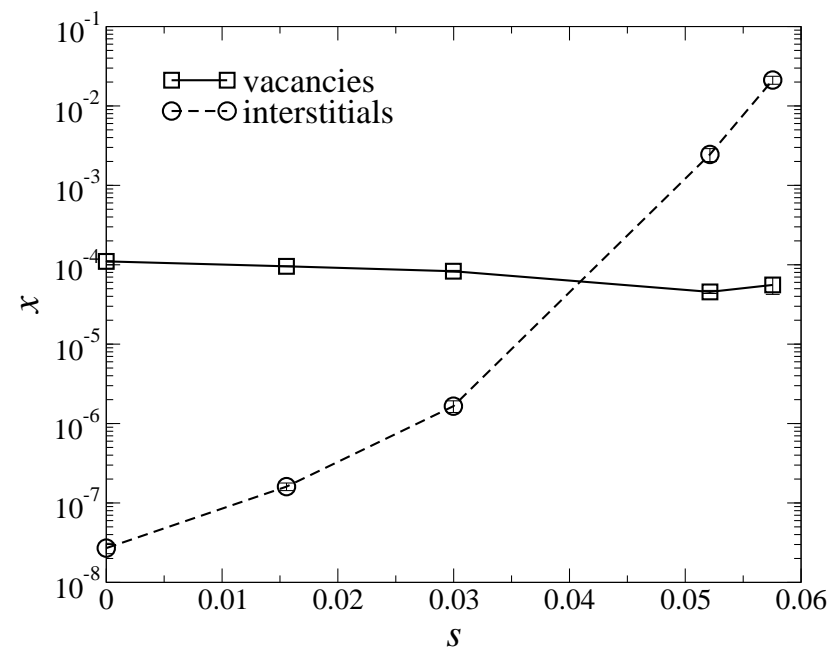

FIG. 1: Point defect concentration $(x)$ versus polydispersity $(s)$

$5.8 \%$. The latter value corresponds to the maximum polydispersity attainable with the chemical potential difference function used. Here, the polydispersity $s$ is defined as the normalized second moment of the particle diameter distribution

$$
s \equiv \frac{\sqrt{\left\langle\sigma^{2}\right\rangle-\langle\sigma\rangle^{2}}}{\langle\sigma\rangle}
$$

All simulations were performed on $256( \pm 1)$ particle systems (a cubic fcc $4 \times 4 \times 4$ lattice); a simulation of a larger system in the monodisperse case in Ref. 5 shows that this particle number is sufficient for the required accuracy. For the (interstitial) calculation of $y_{\text {grow }}$, the $P(a \mid M+1, P, T)$ histograms were divided into 5 windows for which simulations were run in parallel. The multicanonical biasing weights were generated starting with the weights for the monodisperse case and took $10-80$ runs of $4 \cdot 10^{5} \mathrm{MC}$ sweeps (Monte Carlo cycles per particle) per CPU to converge. The final results were obtained using typically 80 runs of $4 \cdot 10^{5}$ sweeps per CPU. In the case of vacancies there was one window for which about 20 runs of $1 \cdot 10^{6}$ sweeps were needed to equilibrate the weights after which about 40 runs of similar length were done for the final results. The equilibrium concentration of the two types of point vacancies as a function of different polydispersities is shown in Table \and Fig. 1

The values of $\mu_{\mathrm{ex}}\left(\sigma_{0}\right)$, required for both the vacancy and interstitial concentration, were calculated using thermodynamic integration using the free energy differentials of Eq. 4 Integration was done along the $P$-v points shown in Table 20 steps between each step and $1 \cdot 10^{6}$ averaging sweeps per step.

\section{DISCUSSION}

The simulation results show a dramatic increase in the interstitial concentration with increasing polydispersity, while the vacancy concentration remains roughly similar over the 


\begin{tabular}{|l|l|l|l|l|l|}
\hline \hline$v$ & 0 & 0.00025 & 0.001 & 0.004 & 0.0056 \\
$P$ & 11.7 & 12.08 & 13.56 & 26.9 & 82.6 \\
\hline$\eta$ & 0.54329 & $0.54522(8)$ & $0.54641(6)$ & $0.55726(6)$ & $0.56997(6)$ \\
$\langle\sigma\rangle$ & 1 & 0.992 & 0.967 & 0.815 & 0.589 \\
$s$ & 0 & $0.015562(3)$ & $0.029974(7)$ & $0.05213(3)$ & $0.05755(5)$ \\
$\mu_{\mathrm{ex}}$ & 17.071 & 17.418 & 18.308 & 24.350 & 37.516 \\
$\mu_{\mathrm{ex}}(\langle\sigma\rangle)$ & 17.1 & 16.9 & 17.8 & 20.1 & 22.5 \\
$\left\langle\sigma_{I}\right\rangle /\langle\sigma\rangle$ & 1 & 0.986 & 0.950 & 0.845 & 0.782 \\
$-\ln P_{\text {ins }}$ & $7.92(1)$ & $8.098(9)$ & $8.77(2)$ & $13.68(4)$ & $26.1(2)$ \\
$y_{\text {grow }}$ & & $32.2(1)$ & $30.8(2)$ & $29.5(2)$ & $40.5(1)$ \\
$x_{V}$ & $1.10(2) \cdot 10^{-4}$ & $9.55(9) \cdot 10^{-5}$ & $8.3(2) \cdot 10^{-5}$ & $4.6(2) \cdot 10^{-5}$ & $5(1) \cdot 10^{-5}$ \\
$x_{I}$ & $2.7(4) \cdot 10^{-8}$ & $1.6(2) \cdot 10^{-7}$ & $1.7(3) \cdot 10^{-6}$ & $2.4(5) \cdot 10^{-3}$ & $2.1(2) \cdot 10^{-2}$ \\
\hline \hline
\end{tabular}

TABLE I: Results for the vacancy and interstitial concentration for the polydisperse hard sphere system. The interstitial concentration for the monodisperse case was taken from Ref. 5. All free energies are in units of $k_{B} T$ and the pressure is in $k_{B} T / \sigma_{0}^{3}$, with the errors in the last digit(s) shown in brackets. Here, $v$ is the polydispersity control parameter (see Eq. 2], $\eta$ is the packing fraction, $\langle\sigma\rangle$ is the mean packing fraction, $s$ is the polydispersity, as defined in Eq. $16\left\langle\sigma_{I}\right\rangle /\langle\sigma\rangle$ is the mean interstitial size relative to the mean particle size, $P_{\text {ins }}$ is the particle insertion probability (see Eq. 15, $y_{\text {grow }}$ is the free energy associated with growing an interstitial (see Eq. 10, $x_{V}$ is the vacancy concentration and $x_{I}$ is the interstitial concentration.

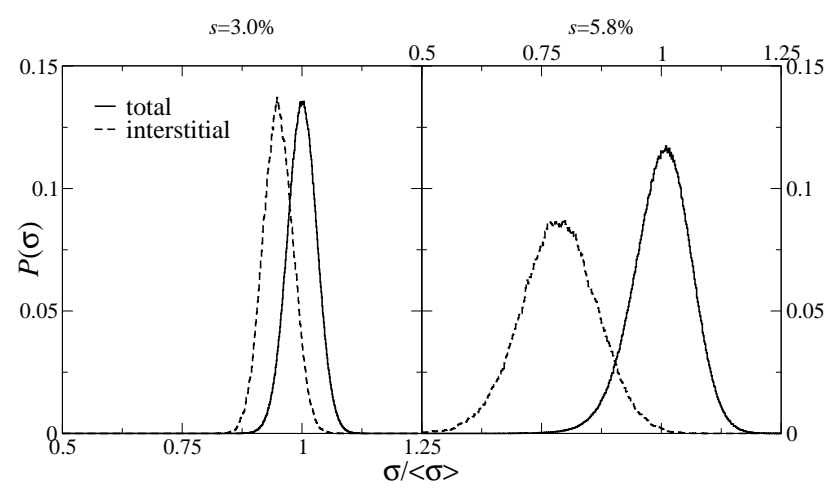

FIG. 2: Normalized size distribution for total system and for the interstitials at polydispersities of $3.0 \%$ (left, $v=0.001$ ) and $5.8 \%$ (right, $v=0.0056$ )

full range of polydispersities. The increase in interstitial concentration can be attributed to the size of the interstitials: if the particle size distribution has non-zero width, the interstitials are smaller than the mean particle size in the crystal, as is shown in Fig. 2

This size difference between interstitials and the surrounding crystal is not an artifact of the simulation method: although the trial moves used in semigrand-canonical simulations are unphysical, the resulting size distribution of interstitials is real. The non-Gaussian particle size distribution in the crystal should be interpreted as a result of fractionation [3, 9] of the coexisting fluid (with the same pressure and polydispersity control parameter $v$ ).

The size distribution of the coexisting fluid is shown in Fig. 3 For small particle sizes, its value is slightly higher than the normal distribution, but at the peak of the interstitial size distribution, the difference in concentration is no more than $7 \%$. To a first approximation, the interstitial concentration in a crystal that has formed from a fluid in which the particle size distribution is exactly Gaussian, should be lower by the same

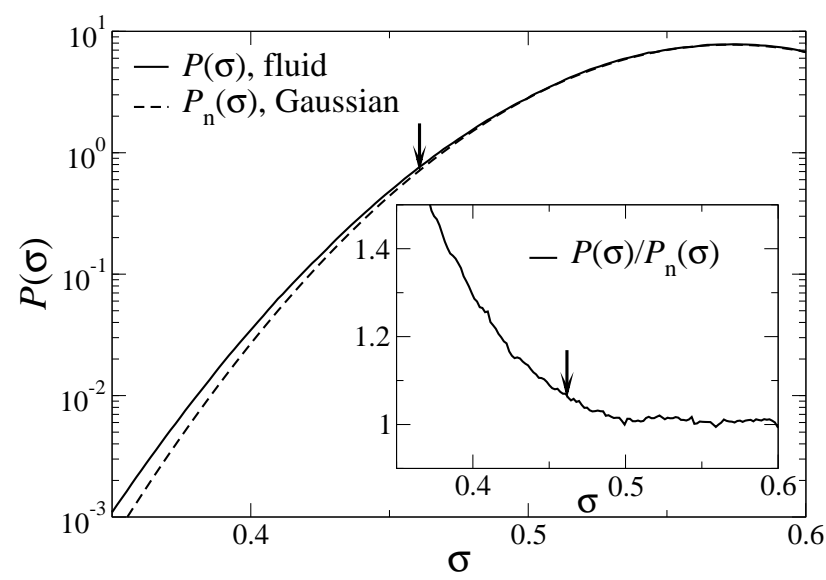

FIG. 3: Particle size distribution of the fluid coexisting with the highest polydispersity solid (solid line, $v=0.0056, s=0.058$ ). The dashed line shows a normal distribution with the same first and second moment. The inset shows the probability distribution relative to the normal distribution. The vertical arrows mark the mean particle diameter of interstitials at the current polydispersity.

amount.

It must be noted, however, that once the crystalline phase starts occupying a sizable fraction of the system volume the size distribution will change and the interstitial concentration will probably be lower. However, the exact size distribution in the crystalline phase is difficult to predict; the size distribution of the fluid itself will change as a result of the growth of the crystalline phase, and because of the high polydispersity of the coexisting fluid, the crystalline phase may be composed of several crystallites, each of which will have its own size distribution.

The influence of the small particles on the interstitial concentration can be illustrated by looking at the free energy of formation of a vacancy as a function of size. If we define a partial interstitial concentration $x_{I}(\sigma)$, we can, as in Eq.7] ex- 


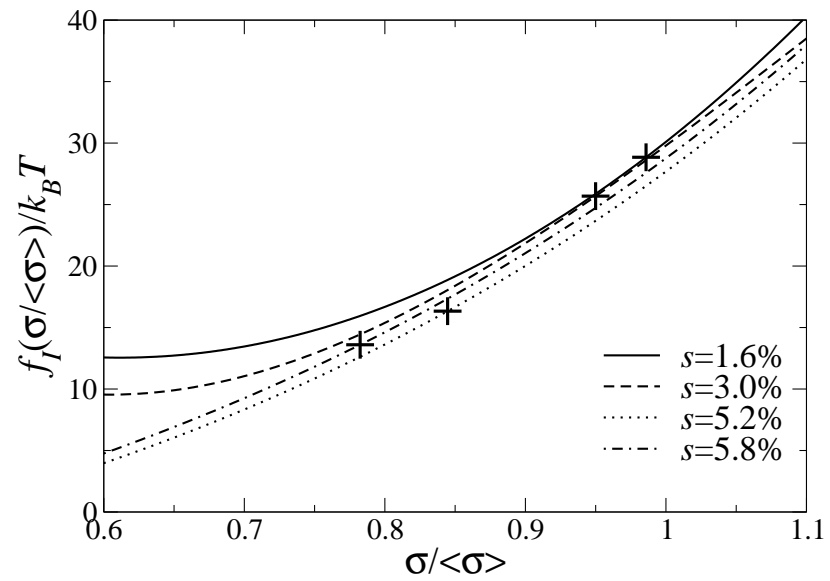

FIG. 4: Interstitial free energy $f_{I}(\sigma)$ for different polydispersities $s$ as a function of renormalized particle size $\sigma /\langle\sigma\rangle$. The $v$ values for the different polydispersities can be found in Table $\square$ The crosses denote the means of the interstitial sizes for the corresponding polydispersities.

press it in terms of the free energy of formation $f_{I}(\sigma)$ and the chemical potential:

$$
x_{I}(\sigma)=\exp \left(-\beta\left[f_{I}(\sigma)-\mu_{\mathrm{ex}}(\sigma)\right]\right)
$$

Assuming that the total interstitial concentration is the integral of the partial concentrations:

$$
x_{I}=\int_{0}^{\infty} d \sigma x_{I}(\sigma)
$$

we can extract $f_{I}(\sigma)$, the free energy associated with creating an interstitial of size $\sigma$, because we know the chemical potential distribution and the partial interstitial concentration. The values for $f_{I}(\sigma)$ at the polydispersities from Table】are shown in Fig 4 To be able to compare values of $f_{I}(\sigma)$ over a large range of $\sigma /\langle\sigma\rangle$, the values for $x_{I}(\sigma)$ in this figure were obtained by fitting the values from the simulations with locally skewed Gaussians

$$
x_{I}(\sigma) \approx a \exp \left[-b\left(\sigma-\left\langle\sigma_{I}\right\rangle\right)^{2}-c\left(\sigma-\left\langle\sigma_{I}\right\rangle\right)^{3}\right]
$$

The fits work very well for the values of $\sigma$ which have been sampled during the simulation, and should yield meaningful results for the range shown in Fig.4

The similarity in slopes and actual values of the $f_{I}(\sigma /\langle\sigma\rangle)$ curves is striking; it means that, for the full range of polydispersities at which a crystal is stable, the partial interstitial concentration depends on the chemical potential distribution and an interstitial free energy which seems to be only weakly dependent on the polydispersity:

$$
f_{I}\left(\frac{\sigma}{\langle\sigma\rangle}\right)=-\kappa\left(\frac{1}{2} \frac{\sigma}{\langle\sigma\rangle}-r_{0}\right)^{2}-f_{I}^{0}
$$

with $\kappa=741 k_{B} T / \sigma_{0}^{2}, r_{0}=0.338 \sigma_{0}$ and $f_{I}^{0}=11.3 k_{B} T$ as fitted parameters from the points in Fig 4 Although the form of this equation was taken from the analytical estimate for the interstitial concentration of Ref. 5, which gives physical meanings to the values of $\kappa$ and $r_{0}$ and has reasonable agreement for $r_{0}$, we stress that, here, $\kappa$ and $r_{0}$ are simply fit parameters.

Because $f_{I}(\sigma /\langle\sigma\rangle)$ hardly depends on the width and, presumably, the shape of the particle size distribution, the small particle tail of the particle size distribution becomes crucial: those particles have the lowest $f_{I}(\sigma /\langle\sigma\rangle)$ and will form the most important contribution to the interstitial concentration. For example, at the near-Gaussian polydispersity of $s=5.2 \%$, obtained by setting $v=0.004$, practically all particles with diameter smaller than $75 \%$ of the mean particle radius are interstitials. This implies that the polydispersity, as measured by the second moment of the particle size distribution in the liquid, is not a good predictor for the interstitial concentration in the solid. The tail of the particle size distribution in the liquid is hard to measure, yet it is all-important for the interstitial concentration.

In the case of vacancies, similar considerations apply in a slightly different form; the vacancy concentration depends on the chemical potential and the free energy of removing a particle while keeping its lattice site. As argued above, they both stay relatively constant at melting for increasing polydispersities which causes the concentration of vacancies to remain roughly similar.

To get an estimate for the interstitial concentration of a colloidal crystal in a suspension, the solely $\sigma$-dependent expression of Eq. 20 must be combined with an estimate for the chemical potential distribution $\mu_{\mathrm{ex}}(\sigma)$, which, in the more conventional ensembles of the experimental situation, does not only depend on the density and the mean particle size, but also on the subsequent moment of the particle size distribution, the polydispersity [9, 10, 11]. An estimate for the absolute values of the chemical potential distribution can be obtained by combining Eq. 2 and the results of table $\square$

In summary, we have shown that for polydisperse hardsphere crystals along the melting curve, the interstitial concentration increases dramatically (going up to $2 \%$ ) while the vacancy concentration remains relatively constant. This can be attributed to the fact that, with increasing polydispersity, there is an increasing probability of finding a particle small enough to have an appreciable probability of fitting in a hole of the underlying crystalline lattice.

This finding has practical implication for the preparation of colloidal crystals from slightly polydisperse solutions. As the presence of interstitials may affect the optical properties of colloidal crystals, it is important to control their concentration. The present calculations show that the interstitial concentration depends sensitively on the tail of the size distribution in the liquid phase. Hence, the polydispersity as such does not provide a reliable criterion to predict interstitial concentrations. Rather, it will be necessary to have an accurate representation of the functional form of the tail of the particlesize distribution (in particular, on the small- $\sigma$ side).

The work of the FOM institute is part of the research program of the Foundation for Fundamental Research on Matter (FOM) and was made possible through financial support by the Dutch Foundation for Scientific Research (NWO). 
[1] A. van Blaaderen, K. P. Velikov, J. P. Hoogenboom, D. L. J. Vossen, A. Yethiraj, R. P. A. Dullens, T. van Dillen, and A. Polman, in Photonic crystals and light organization in the 21st century, edited by C. M. Soukoulis (Kluwer Academic Publishers, Dordrecht, 2001), pp. 239-251.

[2] D. A. Kofke and E. D. Glandt, Mol. Phys 64, 1105 (1988).

[3] P. G. Bolhuis and D. A. Kofke, Phys. Rev. E 54, 634 (1996).

[4] J. M. Polson, E. Trizac, S. Pronk, and D. Frenkel, J. Chem. Phys. 112, 5339 (2000).
[5] S. Pronk and D. Frenkel, J. Phys. Chem. B 105, 6722 (2001).

[6] B. A. Berg and T. Neuhaus, Phys. Rev. Lett. 68, 9 (1991).

[7] G. R. Smith and A. D. Bruce, Phys. Rev. E 53, 6530 (1996).

[8] C. H. Bennett and B. J. Alder, J. Chem. Phys. 54, 4796 (1971).

[9] M. Fasolo and P. Sollich, Phys. Rev. Lett. 91, 068301 (2003).

[10] P. Sollich, J. Phys. Cond. Matt. 14, 1683 (2002).

[11] P. Bartlett, J. Chem. Phys. 109, 10970 (1998). 